\title{
OCORRÊNCIA DE ISOSPORA SP. EM CÃES NA CIDADE dE SÃo PAULO
}

Saemi OGASSAWARA*

Carlos Eduardo LARSSON**

Maria Helena M. A. LARSSON**

Mitika K HAGIWARA **

RFMV-A/17

OGASSAWARA, S.; LARSSON, C.E.; LARSSON, M.H. M.A.; HAGIWARA.M. K. Ocorrência de Isospora sp. em cães na cidade de São Paulo. Rev. Fac. Med. vet. Zootec. Univ. S. Paulo, 15 (2): $-, 137-42,1978$.

RESUMO: O exame coproparasitológico de 167 cães, oriundos de diversas áreas da cidade de São Paulo,de idades, raças e sexos diferentes, revelaram $12(7,2 \%)$ amostras positivas para oocistos de Isospora spp. Estes foram identificados como sendo de I. canis $(3,0 \%)$, I. rivolta $(1,2 \%)$, I. bigemina (1.8\%) e Isospora $s p$. (1,2\%). Entre os cães positivos, $8(66.7 \%)$ eram de faixa etária inferior a 6 meses e $4(39.3 \%)$, superior a 12 meses. Os sintomas clínicos observados nos animais que eliminavam oocistos de isospora nāo foram conclusivos; não se pode tirar qualquer conclusão sobre o papel patogênico desempenhado por aqueles parasitas.

UNITERMOS: Isospora sp, ocorrência; Infecção cāes. *

\section{INTRODUÇÃO}

... Os cães apresentam com relativa freqüência a infecção causada por Isospora spp. Assim é que COSTA \& FREITAS $^{7}$ relataram uma prevalência de $38,88 \%$ de Isospora (I. felis $33,33 \%$ e I. rivolta $5,55 \%$ ) em cães de Belo Horizonte, Minas Gerais; em animais de Porto Alegre, Rio Grande do Sul, FREIRE encontrou $8 \%$ de I. bigemina; AMARAL e cols. ${ }^{.}$verificaram $0,86 \%$ de I. felis na cidade de São Paulo. FERNANDES e cols. ${ }^{12}$ e CHIEFFI e cols. ${ }^{5}$ observaram prevalências de $5,44 \%$ e $3 \%$ de Isospora sp., respectivamente, em Curitiba e Londrina, Paraná. Em Goiânia, Goiás, CARNEIRO e cols. ${ }^{4}$ constataram $4 \%$ de $I$. bigemina.

$\mathrm{O}$ inquérito realizado por LAGE e cols. ${ }^{18}$ revelou $13,5 \%$ de Isospora ( $I$. canis $5,2 \%$ I. rivolta $7,5 \%$ e I. bigemina $0,7 \%$ ) em cães da Guanabara; já FRANKEN e cols ${ }^{14}$ diagnosticaram $24,87 \%$ de animais albergando o protozoário (I. canis $3,04 \%$ e I. rivolta $21,83 \%$ ) no Rio de Janeiro.

No presente trabalho objetivamos estudar a ocorrência de Isospora sp., em cães na cidade de São Paulo, procurando

\footnotetext{
* Professor Assistente Doutor. Departamento de Medicina Veterinária Preventiva e Saúde Animal da Faculdade de Medicina e Veterinaria Zootecnia da USP.

* Professor Assistente. Departamento de Patologia e Clínica Médicas da Faculdade de Medicina Veterinaria e Zootecnia da U.S.P.
} 
também correlacionar os resultados obtidos com os sintomas clínicos observados e à faixa etária.

\section{MATERIAL E MÉTODOS}

Material: no presente estudo foram examinadas 167 amostras de fezes de cães, de diferentes raças, idades e sexos, de diversas áreas da cidade de São Paulo; as fezes eram recebidas junto ao Ambulatório da Disciplina de Patologia e Clínica Médicas de Monogástrico da Faculdade de Medicina Veterinária e Zootecnia da Universidade de São Paulo.

Métodos de exame:

1. A técnica de exame utilizada foi a de centrifugação em água-éter, preconizada por FERREIRA e cols. ${ }^{13}, 1962$.

2. Identificação: a caracterização das espécies de Isospora foi feita após o cultivo dos oocistos em solução aquosa de bicromato de potássio a $2 \%$, à temperatura de 26 - $28^{\circ} \mathrm{C}$. Os oocistos, uma vez esporulados, foram conservados em refrigerador a $4^{\circ} \mathrm{C}$. A mensuração dos oocistos esporulados foi realizada com a ocular micrométrica da Zeiss Wetzlar.

\section{RESULTADOS}

Em 167 amostras de fezes examinadas, $12(7,2 \%)$ revelaram-se positivas para a presença de Isospora spp. Estas foram identificadas segundo suas características estruturais.

A Isospora identificada como $I$. canis apresentou oocistos de formas ovóides, de paredes lisas, sem micrópila, com ausência de grânulo polar e corpúsculo residual oocístico. Os esporocistos apresentaram-se elipsóides, sem corpúsculo de Stieda, com presença de corpúsculo residual esporocístico. As dimensões de 44 oocistos esporulados foram de $31,5-40,7 \mu \mathrm{m}$ de comprimento e $25,9-33,3 \mu \mathrm{m}$ de largura, com uma dimensão média de $31,0 \times 37,0-\mu \mathrm{m}$. Os 88 esporocistos mediram de $14,8-24,1$ $\mu \mathrm{m}$ de comprimento e $12,9-18,5 \mu \mathrm{m}$ de largura, com a dimensão média de $15,4 \mathrm{X}$ $20,4 \mu \mathrm{m}$.
Isospora rivolta: os oocistos apresentaram-se ovóides, de paredes lisas, sem micrópila e com ausência de corpúsculo residual oocístico; os esporocistos eram elipsóides, sem corpúsculo de Stieda e com a presença de corpúsculo residual esporocístico. As dimensões de 100 oocistos esporulados foram de 18,5 $-27,8 \mu \mathrm{m}$ de comprimento e $16,7-$ $25,9 \mu \mathrm{m}$ de largura, com a dimensão média de $21,3 \times 23,4 \mu \mathrm{m}$. As dimensões de 155 esporocistos foram de $9,3-18,5$ $\mu \mathrm{m}$ de comprimento e $7,4-11,1 \mu \mathrm{m}$ de largura, com a dimensão média de 10,7 x $14,0 \mu \mathrm{m}$.

Isospora bigemina: os oocistos apresentaram-se esféricos, de paredes lisas, sem micrópila, sem corpúsculo residual oocístico; os esporocistos sem corpúsculos de Stieda e com corpúsculo residual esporocístico. As dimensões de 100 oocistos esporulados foram de $9,3-$ $14,8 \mu \mathrm{m}$ de comprimento e $7,4-13,0 \mu \mathrm{m}$ de largura e com a a dimensão média de $10,9 \times 11,4 \mu \mathrm{m}$. A mensuração de 100 esporocistos forneceram os seguintes dados: $5,6-9,3 \mu \mathrm{m}$ de comprimento, $3,7-7,4 \mu \mathrm{m}$ de largura e uma dimensão média de 5,7 x 7,1 $\mathrm{\mu m}$.

Nas TABELAS I, II, III, e IV figuram os resultados obtidos.

Na TABELA I foram relacionados o número e a porcentagem de animais positivos em função das diferentes espécies de Isospora; nas TABELAS II e III foram dispostas, respectivamente, as dimensões dos oocistos esporulados e dos esporocistos de Isospora spp.

Finalmente, na TABELA IV foram relacionados a raça, a idade em meses, o sexo dos animais, bem como o diagnóstico clínico e resultado dos exames coproparasitológicos.

\begin{tabular}{|c|c|c|}
\hline Isospora & $\underset{N^{\circ}}{\text { Animais }}$ & $\begin{array}{c}\text { positivos } \\
\%\end{array}$ \\
\hline $\begin{array}{l}\text { I.bigemina } \\
\text { I.rivolta } \\
\text { I.canis } \\
\text { Isospora sp. }\end{array}$ & $\begin{array}{l}3 \\
2 \\
5 \\
2\end{array}$ & $\begin{array}{l}1,8 \\
1,2 \\
3,0 \\
1,2\end{array}$ \\
\hline Total & 12 & 7,2 \\
\hline
\end{tabular}


TABELA II. - Dimensões dos oocistos esporulados de Isospora spp.

\begin{tabular}{|l|c|c|c|}
\hline \multicolumn{1}{|c|}{ Isospora } & $\begin{array}{c}\text { Comprimento } \\
(\mu \mathrm{m})\end{array}$ & $\begin{array}{c}\text { Largura } \\
(\mu \mathrm{m})\end{array}$ & $\begin{array}{c}\text { Média } \\
(\mu \mathrm{m})\end{array}$ \\
\hline I.bigemina & $9,3-14,8$ & $7,4-13,0$ & $10,9 \times 11,4$ \\
I.rivolta & $18,5-27,8$ & $16,7-25,9$ & $21,3 \times 23,4$ \\
I.canis & $31,5-40,7$ & $25,9-33,3$ & $31,0 \times 37,0$ \\
\hline
\end{tabular}

TABELA III. - Dimensōes dos esporocistos de Isospora spp.

\begin{tabular}{|l|c|c|c|}
\hline Isospora & $\begin{array}{c}\text { Comprimento } \\
(\mu \mathrm{m})\end{array}$ & $\begin{array}{c}\text { Largura } \\
(\mu \mathrm{m})\end{array}$ & \multicolumn{1}{c|}{$\begin{array}{c}\text { Média } \\
(\mu \mathrm{m})\end{array}$} \\
\hline $\begin{array}{l}\text { I.bigemina } \\
\text { l.rivolta } \\
\text { I.canis }\end{array}$ & $5,6-9,3$ & $3,7-7,4$ & $5,7 \times 7,01$ \\
\end{tabular}

TABELA IV - Diagnóstico clínico e resultado dos exames coproparasitológicos em cães de diferentes raças, sexos e idades.

\begin{tabular}{|c|c|c|c|c|c|c|}
\hline $\begin{array}{c}\text { Caso } \\
n^{\circ}\end{array}$ & Raça & Sexo & Idade & Diagnóstico Clínico & \multicolumn{2}{|c|}{$\begin{array}{c}\text { Exame Coproparasitológico } \\
\text { Helmintos }\end{array}$} \\
\hline 1 & Pinscher & $\mathrm{M}$ & $15 \mathrm{~m}$ & Cinomose & Ancylostoma sp & Isospora bigemina \\
\hline 2 & Poodle & $\mathrm{M}$ & $5 \mathrm{~m}$ & & Negativo & Isospora rivolta \\
\hline 3 & SRD & $\mathrm{M}$ & $36 \mathrm{~m}$ & Gastroenterite aguda & Ancylostoma sp & Isospora sp \\
\hline 4 & SRD & $\mathrm{M}$ & $5 \mathrm{~m}$ & Enterite hemorrágica & Trichuris sp & Isospora sp \\
\hline 5 & $\begin{array}{c}\text { Pastor } \\
\text { Alemão }\end{array}$ & $\mathrm{M}$ & $24 \mathrm{~m}$ & Ascite hipoproteinêmica & $\begin{array}{c}\text { Ancylostoma sp } \\
\text { Trichuris sp }\end{array}$ & Isospora bigemina \\
\hline 6 & $\begin{array}{c}\text { Pastor } \\
\text { Alemão }\end{array}$ & $\mathrm{M}$ & $14 \mathrm{~m}$ & Cinomose & Negativo & Isospora bigemina \\
\hline 7 & Boxer & $\mathrm{M}$ & $2 \mathrm{~m}$ & Enterite hemorrágica & Ancylostoma sp & Isospora canis \\
\hline 8 & SRD & $\mathrm{F}$ & $2 \mathrm{~m}$ & Gastroenterite & Ancylostoma sp & Isospora canis \\
\hline 9 & SRD & $\mathrm{F}$ & $3 \mathrm{~m}$ & $*$ & $\begin{array}{c}\text { Ancyl.sp Tox.sp } \\
\text { Trichuris sp }\end{array}$ & Isospora canis \\
\hline 10 & SRD & $\mathrm{M}$ & $3 \mathrm{~m}$ & $*$ & $\begin{array}{c}\text { Toxocara sp } \\
\text { Ancylostoma sp }\end{array}$ & Isospora rivolta \\
\hline 11 & SRD & $\mathrm{M}$ & $3 \mathrm{~m}$ & & $\begin{array}{c}\text { Toxocara sp } \\
\text { Ancylostoma sp }\end{array}$ & Isospora canis \\
\hline 12 & SRD & $\mathrm{F}$ & $3 \mathrm{~m}$ & $*$ & $\begin{array}{c}\text { Ancyl.sp Tox.sp } \\
\text { Trichuris sp }\end{array}$ & Isospora canis \\
\hline
\end{tabular}

$M=$ Macho; $F$ = Fêmea; SRD = Sem Raça Definida; "Assintomático $m$ = meses

\section{DISCUSSÃO}

Investigações recentes têm demonstrado que coccídias de felídios são biologicamente distintas daquelas de canideos $^{(8),(9),(11),(20)(22),(25)}$. Assim, ficou patente pelos trabalhos de NEMESE$\mathrm{RI}^{22}$ e $\mathrm{SHAH}^{25}$ / que a despeito da semelhança estrutural a I.felis do gato é distinta da $I$. felis dos cães, recebendo, portanto, esta última a denominação de I.canis. Ficou também demonstrado através de infecções experimentais que I.rivolta do gato é distinta de I.rivolta encontrada parasitando cães. Em vista desses resultados DUBEY ${ }^{9}$ propôs, para I.rivolta dos cães, uma nova denominação-I. ohioensis. $\mathrm{O}$ citado autor justifica-se afirmando que a I.rivolta foi inicialmente descrita nos gatos e, posteriormente, nos cães.

Muita polêmica existe acerca da I.bigemina (Stiles, 1891) Lühe, 1906. Esta espécie foi, primeiramente, denominada de Coccidium bigeminum (Stiles, 1891) em função do encontro de oocistos, com dois esporocistos, nas vilosidades intestinais de cães. Segundo Stiles 
(1891),esta espécie correspondia ao organismo descrito por FINCK (1854) nas vilosidades intestinais de gato e descrito, posteriormente, nos cães por VIRCHOW (1860), LEUCKART (1860) e por RIVOLTA (1878). O último autor denominou-o de Cytospermiun villorum intestinalium canis ${ }^{30,31,32}$.

$\mathrm{Na}$ realidade, dois tipos de I.bigemina haviam sido relatados na espécie canina. $\mathrm{O}$ primeiro deles, cujos oocistos eram pequenos ${ }^{19}$ com desenvolvimento em células epiteliais do intestino delgado e que eram elimiminados nas fezes sob a forma não esporulada; o segundo, I. bigemina de oocistos grandes ${ }^{19}$, com desenvolvimento ao nível da lâmina própria e que eram eliminados já esporulados nas fezes.

Os dois tipos de $I$. bigemina que têm sido encarados como sendo uma única espécie são, à luz dos conhecimentos atuais, dois parasitas distintos ${ }^{10.19}$. Mas, como a denominação $I$. bigemina foi utilizada, pela primeira vez, para aquela do tipo grande (Stiles, 1891), o agora conhecido como Sarcocystis bigemina (Stiles, 1891) ${ }^{19}$, ou Sarcocystis spp., tem sido propostas, para o tipo pequeno de $I$. bigemina dos cães, as seguintes denominações: $I$. wallacei e $I$. heydorni, respectivamente, por DUBEY ${ }^{10} \mathrm{e}^{\text {TADROS e LAARMAN }}{ }^{28}$. LEVINE ${ }^{9}$ havia designado Isospora n.sp. Dubey, mas modificou-a para I.heydorni após a publicação de TADROS e LAARMAN ${ }^{28}$.

A I. bigemina descrita nos gatos, cujos oocistos eram eliminados sob forma não esporulada, foi designada por LEVINE ${ }^{19}$, Toxoplasma gondii, ou Toxoplasma hammondi e/ou Besnoitia spp. A semelhança do que foi mencionado para os cães, outras I. bigemina com oocistos esporulados já ao nível da lâmina própria são encontradas nos gatos e são atualmente catalogadas como Sarcocystis spp. No passado, a denominação I. bigemina foi empregada para, pelo menos, 9 coccídias distintas de felídeos e 7 de canídeos ${ }^{10}$.

No presente trabalho foram identificadas 3 espécies de Isospora, de conformidade com os dados constantes das TABELAS I, II e III, porquanto as dimensões dos oocistos e esporocistos, bem como outras características morfológicas, foram concordantes com as observadas por outros autores 20,23.2. As espećies encontradas foram: I. canis (sin. Ifelis, Wenyon, 1923), I. ohioensis (sin. I. rivolta - Grassi, $1879-\mathrm{We}-$ nyon, 1923) e Isosporan. sp. (sin. II. bigemina (Stiles, 1891) Lühe, 1906).

Com o escopo de permitir uma melhor comparação dos resultados deste e de outros trabalhos nacionais, procuramos, no presente relato, manter as antigas denominações.

As três Isospora observadas por nós têm sido assinaladas no Brasil com a predominância de uma ou outra ou com a ocorrência de uma ou mais espécies, em função da região. Assim, a I.bigemina já foi assinalada em São Paulo ${ }^{2}$, Goiás ${ }^{4}$, Bahia ${ }^{21}$, Rio Grande do Sul ${ }^{6}, 16,{ }^{17}$ e Rio de Janeiro $^{18}$; a I. canis, por sua vez, foi observada em São Paulo ', Minas Gerais 7, Bahia ${ }^{26}$, Rio Grande do Sul ${ }^{15}$ e Rio de Janeiro ${ }^{14},{ }^{18} \mathrm{e}$, finalmente, a $I$. rivolta foi descrita em São Paulo ${ }^{3}{ }^{24}$, Minas Gerais 7, Rio de Janeiro ${ }^{14}{ }^{18}$ e Pernambuco ${ }^{29}$. No Paranás, ${ }^{12}$, tem sido relatada a presença de Isospora, mas sem identificação de espécie(s).

Através a análise da TABELA I, pode-se constatar a não-observância no presente trabalho, de infecções múltiplas por várias espécies de Isospora num mesmo animal. Deve-se salientar, ainda, que a Isospora sp. na citada TABELA I, não identificada por motivos alheios à nossa vontade, era, provavelmente I.canis.

Observando a TABELA IV, podese notar que dos 12 animais positivos, 8 $(66,7 \%)$ eram de faixa etária inferior a 6 meses e $4(39,3 \%)$ superior a 12 meses, resultados estes concordantes com aqueles de CHIEFFI e cols. ${ }^{3}$, e SOIFER ${ }^{27}$. CHIEFFI e cols. ${ }^{5}$, no Paraná, encontraram $80 \%$ de ocorrência em animais com menos de 12 meses de vida. Do mesmo modo SOIFER ${ }^{27}$, em Houston, observou $86 \%$ de animais positivos para Isospora, na mesma faixa etária. Tais achados vêm comprovar que, de fato, animais jovens são mais suscetiveis à infecção do que os adultos e, portanto, o fator idade desempenha importante papel na epidemiologia da isosporíase. 
A ação patogênica do protozoário em apreço, nos carnívoros domésticos, constitui, ainda, assunto bastante controvertido ${ }^{10}{ }^{27}$. Pela análise da TABELA IV pode-se verificar que 2 animais (casos $\mathrm{n}^{\circ} 2$ e 6) apresentaram apenas Isospora em suas fezes, e os demais foram positivos para uma a três espécies diferentes de helmintos, bem como manifestaram outros quadros mórbidos de etiologia diversa. Por esta razão, torna-se bastante difícil estabelecer a patogenicidade das várias espécies de Isospora de per si, em condições naturais de criação e manejo, havendo, pois, a necessidade de estudos em condiçōes experimentais adequadamente controladas.

\section{RFMV-A/17}

OGASSAWARA. S.; LARSSON, C.E.; LARSSON, M.H.M.A.; HAGIWARA, M.K

Incidence of Isospora $s p$. in dog of the city of São Paulo.

Rev. Fac. Med. vet. Zootec. Univ.S.Paulo, 15 (2):-, 137-42, 1978

SUMMARY: The examination of $167 \mathrm{fecal}$ samples from dogs from different areas of the city of São Paulo, of different ages, races and both sexes, revealed $12(7,2 \%)$ positive samples for Isesporaspp.oocysts. They were identified as I.canis(3,0\%).I.rivolta $(1,2 \%)$,I.bigemina $(1,8 \%)$ e Isospora sp. (1,2\%). Among positive animals, $8(66,7 \%)$ were under 6 month-old age and 4 $(39,3 \%)$ over 12 month-old age. Clinical symptoms observed in the animals that yielded isosporan oocysts were not definitive; thus no conclusion could be obtained about the pathogenic role played by the parasites.

UNITERMS: Isospora sp.incidence *; Infection., dogs.

\section{REFERÊNCIAS BIBLIOGRÁFICAS}

1-AMARAL, V.; AMARO, R.G.; BIRGEL, E.H. - Sobre a presença de Isospora felis Wenyon, 1923 (Protozoa:Eimeriidae Poche, 1913) em Canis familiaris, em São Paulo. Arq. Inst. Biol., São Paulo, 3/(3): 101-2,1964.

2 - AMARAL, V. \&; BIRGEL, E.H. Nota sobre a ocorrência da Isospora bigemina (Stiles, 1891) Lühe, 1906 em Canis familiaris, em São Paulo e distribuição geográfica das espécies de Isospora em cães e gatos, no Brasil. Arq. Inst. Biol., São Paulo, 35(2): 77-81,1968.

3 - AMARAL, V. ; REBOUÇAS, M.M.; BIRGEL, E.H. Observações so bre a Isospora rivolta (Grassi, 1879) Wenyon, $1923 \mathrm{em}$ Canis familiaris de São Paulo. Rev. Med. vet.,3(1): 69$73,1967$.

4 - CARNEIRO, JR.; PEREIRA,E.; PINHEIRO,Z.B. Isospora bigemina Stiles, 1891 em Canis familiaris de Goiânia. Rev. Pat. Trop.,2(3) : 217-9,1974.

5 - CHIEFFI,P.P.; MÜLLER, E.E.; VIOTTI,N.M.A.; MORETTI,I.G. Estudo sobre a prevalência de enteroparasitas em cães da zona urbana do Município de Londrina, Estado do Paraná, Brasil. Científica,4 (1): 64-7,1976.

6 - CORREA.O. \& BANOLAS,G. Um caso de isosporose canina por Isospora bigemina e Isospora rivolta. Rev. Fac.Agron. Vet. Porto Alegre, 9: 435,1968 .
7 - COSTA, H.M.A. \& FREITAS,M.G. Isospora felis Wenyon, 1923 e Isospora rivolta Grassi, 1879, em cães de Belo Horizonte. Arq. Esc.Sup.Vet., 12: 127-30,1959.

8 - DUBEY, J.P. Experimental Isospora canis and Isospora felis infection in mice, cats, and dogs. J.Protozool.,22 (3) : 416-7,1975.

9- DUBEY, J.P. Isospora ohioensis sp.n proposed for I.rivolta of the dog. J.Parasitol., 61 (3): 462-5,1975.

10 - DUBEY, J.P. A review of Sarcocystis of domestic animals and of other coccidia of cats and dogs. J. Amer. vet.med. Ass., 169 (10): 1061-78, 1976.

11 - DUBEY, J.P.; MILLER, N.L.; FRENKEL,J.K. The Toxoplasma gondii oocyst from cat feces. J.Exp.Med.,132: $636-62,1970$.

12 - FERNANDES, B. F.; ROESEL, M.P. NASCIMENTO, E.E.; SCHULTZ, J.A. Considerações sobre parasitas gastrointestinais de cão (Canis familiares), diagnosticados em exame de fezes. Arq. Biol.Tecnol., 16: 126-8, 1973.

13 - FERREIRA,L.F.; MORTEO,R.E.; SILVA,J.R. Padronização de técnicas para exame parasitológico das fezes. J.bras. Med., 6: 241-57, 1962.

14 - FRANKEN,A.M.J.; FIGUEIREDO,M.J. COUTINHO, S.G. Prevalência de Isospora em cães de diferentes áreas 
da cidade do Rio de Janeiro. Rev. Soc. Bras.Med.Trop.9 (1): 43-51, 1975.

15 - FREIRE,J.J. Isosporose canina. Isospora felis Wenyon, 1923. Bol.Dir.Prod.An., Porto Alegre, 2 (4): 30-4, 1946.

16 - FREIRE,J.J. Isosporose canina por Isospora bigemina (Stiles, 1891).Rev.Fac. Agron. Vet., Porto Alegre, 5: 177 86, 1962.

17 - GONÇALVES,P.C. Sobre um caso de Isosporose canina, ocorrido em nosso meio. Rev.Esc.Agron.Vet., Porto Alegre, 1 (3): 47-50, 1956.

18 - LAGE,H.A.; LAGE,S.A.; LOBO,M.E Cross - infection experiments with Isospora of cats and dogs. Mem.Inst. Oswaldo Cruz, 72 (1/2): 137-42, 1974.

19 - LEVINE N.D. Nomenclature of Sarcocystis in the ox and sheep and of fecal coccidia of the dog and cat. J.Parasitol., 63 (1): 36-51, 1977

20 - LEVINE, N.D. \& IVENS, V. Isospora species in the dog. J. Parasitol.,51 (5): 859-64, 1965.

21 - MOURA COSTA, M.D. Isosporose do cão com a descrição de uma nova variedade (Isospora bigemina, Stiles, 1891 bahiensis n. Var.). Bol.Inst.Biol. da Bahia, Salvador, 3: 107-12, 1956.

22 - NEMESÉRI, L. Beitrage Zur Atiologie der Coccidiose der Hunde I. Isospora canis sp.n. Acta Vet Acad.Sci.Hung., 10: $95-9,1960$

23 - PELLÉRDY, L.P. Coccidia and Coccidiosis. 2 and. Ed., Paul Parey, Federal Republic of Germany, 1977.

24 - PINTO, C. \& VALliN, A.R. Estudos sobre Coccideas. Bol. Inst. bras.Sci. 2 (6): 216-9, 1926
25 - SHAH,H.L. Isospora species of the cat and attempted transmission of $I$. felis, Wenyon, 1923 from the cat to the dog. J.Protozool., 17 (4): 603-9, 1970.

26 - SILVA, A.M.; BASTOS, W.D. A.; SANTOS, M.D. Ocorrência de Isospora felis Wenyon, 1923 parasitando caninos de Salvador - Bahia Brasil. Bol.Inst.Biol. da Bahia, Salvador, 12 (1): 23-5, 1973.

27-SOIFER. F.K. Intestinal parasitism. In: Kirk,R.W. Current veterinary therapy. W.B. Saunders Co., 1977. v.6,p.95866.

28 - TADROS,W. \& LAARMAN.J.J. Sarcocystis and related coccidian parasites: a brief general review, together with a discussion on some biological aspects of their life cycles and a new proposal for their classification. Acta Leidensia, 44: 1-107, 1976.

29 - TORRES, S. Isospora rivoltae (Grassi, 1879) em cães de Pernambuco. Rev. Soc. paul. Med. Vet., 5 (2): 61-3, 1938.

30 - WENYON, C.M. Coccidiosis of cats and dogs and the status of the Isospora of man. Ann.Trop. Med. Parasitol.,17 (2): 231-88, 1923.

31 - WENYON, C.M. Coccidia of the genus Isospora in cats, dogs and man. Parasitology, 18 (3): 253-66, 1926

32 - WENYON, C.M. Protozoology. A manual for medical men, veterinarians and zoologists. London, Baillière, Tindall \& Cox, 1926.

Aprovado para publicação em 4-9-1978 